\title{
Calcineurin inhibitors target Lck activation in graft-versus-host disease
}

\author{
Nicole M. Carter and Joel L. Pomerantz \\ Department of Biological Chemistry and Institute for Cell Engineering, The Johns Hopkins University School of Medicine, Baltimore, Maryland, USA.
}

\begin{abstract}
Calcineurin inhibitors (CNIs) such as cyclosporin A and FK506 are widely administered immunosuppressive drugs. Calcineurin relieves inhibitory phosphorylation from nuclear factor of activated T cells (NFAT) transcription factors downstream of $\mathrm{T}$ cell receptor engagement, resulting in their nuclear translocation and the production of cytokines, including IL-2, IFN- $\gamma$, and TNF- $\alpha$. It was previously believed that CNIs downregulate immunity by reducing NFAT activation. However, work from Otsuka et al. in this issue of the $\mathrm{JCl}$ revealed a second mechanism by which CNIs suppress $T$ cell function. The authors previously reported that calcineurin removes an inhibitory phosphate from the tyrosine kinase Lck at Ser59 (Lck-S59) and that this dephosphorylation positively regulates $\mathrm{T}$ cell activation. In the present work, the authors showed that inhibition of Lck-S59 dephosphorylation was essential for the CNI-mediated suppression of acute graft-versus-host disease (aCVHD). These findings have important implications for future approaches to the management of aCVHD, organ transplant rejection, and autoimmune disease.
\end{abstract}

\section{Calcineurin inhibitors} and their toxicity

Calcineurin inhibitors (CNIs), including cyclosporin A (CsA) and FK506, are widely prescribed for the management of solid organ transplant rejection (1) and acute graft-versus-host disease (aGVHD), a severe complication of hematopoietic stem cell transplantation in which donor $\mathrm{T}$ cells attack recipient tissues (2-4). CNIs have also been used to treat autoimmune diseases, including lupus nephritis, autoimmune membranous nephropathy, and rheumatoid arthritis (5). Despite the clinical utility of CNIs, their use is not without drawbacks. For example, CNIs can cause acute nephrotoxicity, diabetes, electrolyte disturbances (1), neurotoxicity (6), and hypertension (7). These side effects possibly occur because the expression of calcineurin and one of its major targets, nuclear factor of activated T cells (NFAT), extends beyond immune cells $(5,8)$. Therefore, there is substantial interest in developing targeted alternatives to existing CNIs that maintain their immunosuppressive function but with fewer off-target effects.

\section{Calcineurin functions in T cells}

Calcineurin is a $\mathrm{Ca}^{2+}$-calmodulin-dependent serine-threonine phosphatase that is activated downstream of $\mathrm{T}$ cell receptor (TCR) engagement. A major target of calcineurin in T cells is the NFAT family of transcription factors, particularly NFAT1 and NFAT2. NFAT proteins are constitutively phosphorylated at multiple serine and threonine residues, which maintain NFAT in the cytoplasm. Removal of these phosphate residues by activated calcineurin allows NFAT to localize to the nucleus to induce NFAT-dependent gene

\section{Related Article: https://doi.org/10.1172/JCl147683}

transcription (9). NFAT activation is crucial for the production of cytokines, including IL-2, IL-4, IL-10, IFN- $\gamma$, and TNF- $\alpha$, as well as the upregulation of activation markers CD4OL and FasL (9). CNIs form complexes with cytosolic immunophilins, which then bind to calcineurin and inhibit its phosphatase activity, thereby inhibiting NFAT activation (10).

In addition to NFAT, previous work from the Ashwell lab recently revealed a second important target of calcineurin in T cells (11). Lck is a Src family tyrosine kinase that is recruited to the TCR and activated immediately downstream of TCR engagement. Lck phosphorylates tyrosine-based activation motifs in the TCR CD3 chains, resulting in the binding and activation of the tyrosine kinase ZAP70 (12). ZAP-70 then phosphorylates adaptors, including LAT and SLP-76, leading to the recruitment of additional adaptors and signaling molecules and allowing for full $\mathrm{T}$ cell activation (13). In activated $\mathrm{T}$ cells, ERK phosphorylates Lck at Ser59 (14). Lck phosphorylation at Ser59 interferes with interactions between Lck and its substrates, although the effect of this phosphorylation was, until recently, controversial (12). The Ashwell lab's prior work unveiled that downstream of TCR engagement, calcineurin is recruited to the TCR, where it dephosphorylates Lck-S59 (11). In human Jurkat $\mathrm{T}$ leukemia cells, mutation of Lck-S59 to Ala, which cannot accept a phosphate, resulted in increased phosphorylation of ZAP-70, LAT, ERK, and p38 and increased production of IL-2, whereas mutation to Glu, a phosphomimetic, had the opposite effects (11). Importantly, the previous study also found that dephosphorylation of Lck-S59 by calcineurin promotes adhesions between the $\mathrm{T}$ cell integrin LFA-1 and ICAM-1, which is expressed by endothelial cells and antigen presenting cells. Treatment with CsA or FK506 inhibited dephosphorylation of Lck-S59 and resulted in decreased LFA-1-ICAM-1 adhesions (11). 


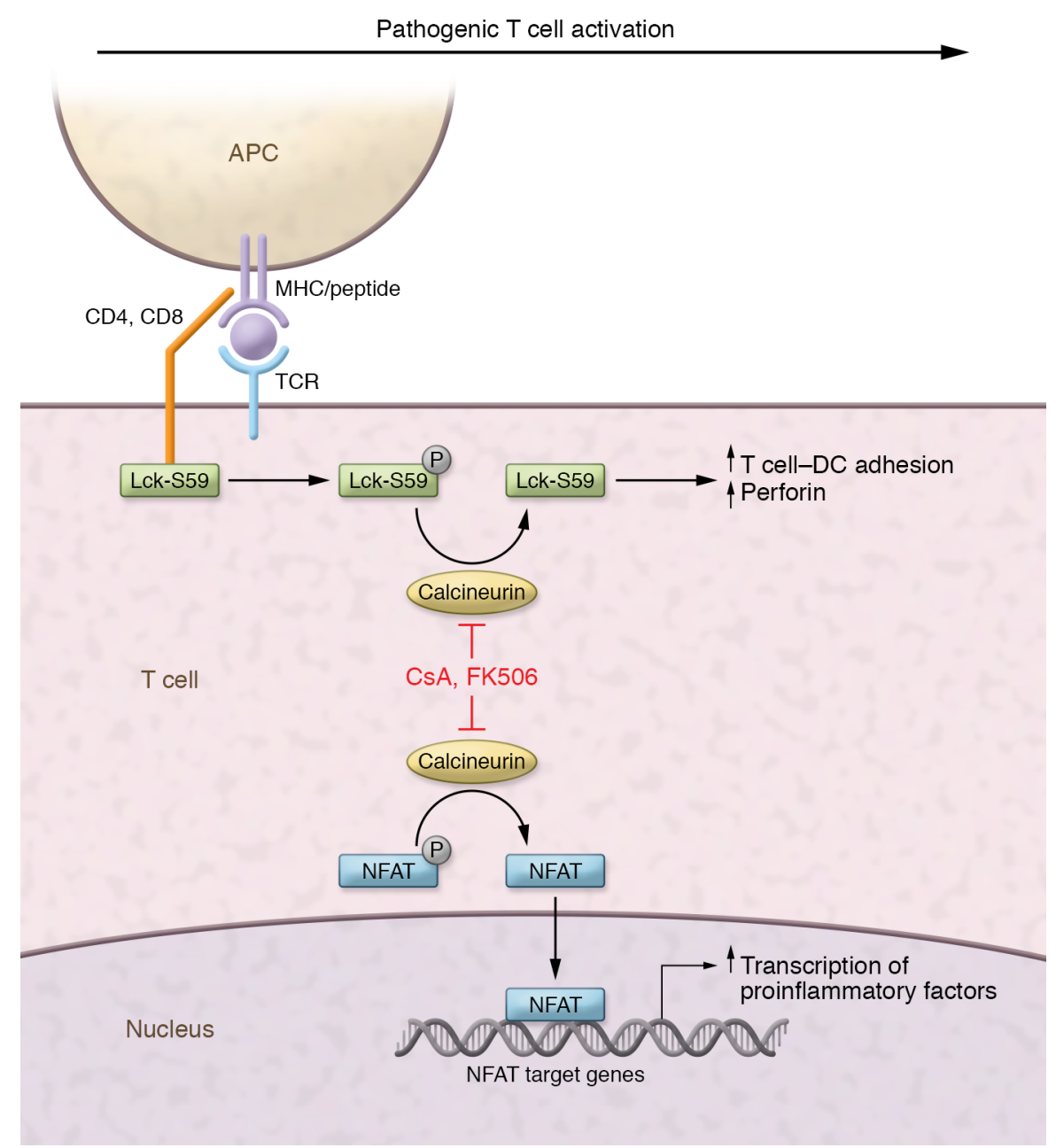

\section{A second mechanism of CNI-mediated immunosuppression}

It has long been assumed that the immunomodulatory effect of CNIs is due to inhibition of NFAT signaling. However, the discovery that calcineurin also dephosphorylates Lck-S59 at the TCR, promoting $\mathrm{T}$ cell activation and LFA-1-mediated adhesions, raises the question of whether the inhibition of Lck-S59 dephosphorylation contributes to immunosuppression by CNIs, and if so, how. In this issue of the JCI, Otsuka et al. addressed these questions and found that inhibition of Lck-S59 dephosphorylation was critical for the control of aGVHD by CNIs (15). The authors studied a mouse model in which Lck-S59 was mutated to Ala (Lck-S59A; ref. 16). As in Jurkat $\mathrm{T}$ cells, treatment of WT mouse primary $\mathrm{T}$ cells with CsA inhibited TCR-proximal signaling events, including the phosphorylation of ZAP-70 and LAT, whereas these events were unaffected by CsA treatment
Figure 1. A secondary NFAT-independent model by which $\mathrm{CNIs}$ suppress pathogenic $\mathrm{T}$ cell activation. TCR-proximal calcineurin dephosphorylates Lck-S59, which specifically promotes the formation of T/DC adhesions and T cell cytotoxicity via the upregulation of perforin. CNIs, such as CsA and FK506, block Lck dephosphorylation, and this action on Lck is essential for control of aGVHD.

that CsA treatment reduced the number of liver-infiltrating regulatory $\mathrm{T}$ cells (Tregs) in mice receiving either WT or Lck-S59A donor cells, and they speculated that reduced Tregs could explain why CsA treatment worsened disease in mice receiving Lck-S59A donor cells. CsA treatment also failed to reduce the number of donor Lck-S59A CD4 ${ }^{+}$and $\mathrm{CD}^{+}{ }^{+} \mathrm{T}$ cells infiltrating the liver, whereas CsA treatment substantially reduced the liver infiltration of WT donor T cells. Importantly, markers of NFAT activation were similarly reduced in WT and Lck-S59A donor T cells following CsA treatment. These data indicate that inhibition of TCR-proximal signaling is an important mechanism by which CNIs alleviate aGVHD and that inhibition of NFAT signaling alone is insufficient to control disease (15).

Finally, Otsuka et al. investigated two specific $\mathrm{T}$ cell functions that are regulated by dephosphorylation of Lck-S59. Expanding on the previous finding that calcineurin promotes LFA-1-mediated adhesions (11), the authors examined the effect of CsA on adhesions between $\mathrm{CD}^{+}$or $\mathrm{CD}^{+} \mathrm{T}$ cells and antigen-pulsed dendritic cells (DCs). CsA reduced T/DC adhesions for WT CD $4^{+}$and $\mathrm{CD}^{+}$T cells but not for LckS59A cells. In vivo, CsA treatment reduced the number and size of antigen-specific $\mathrm{T} /$ DC clusters in the lymph nodes for WT T cells but not for Lck-S59A cells, suggesting that calcineurin must dephosphorylate Lck-S59 for optimal antigen-specific T/ DC clustering to occur. The authors also examined the effect of CsA treatment on cytotoxicity and found that CsA inhibited the expression of perforin, which punctures target cell membranes, in WT but not Lck-S59A cells. The differential effect on perforin expression indicates that Lck-S59 dephosphorylation by calcineurin regulates cytotoxicity independently of NFAT (Figure 1 and ref. 15). 


\section{Implications and potential future directions}

Notably, Otsuka et al. determined that a widely used class of drugs causes immunosuppression through a previously unappreciated mechanism (15). Furthermore, the fact that inhibition of TCR-proximal signaling by CNIs was essential for the control of aGVHD raises the question of whether inhibition of TCR-proximal signaling is sufficient to control aGVHD or if NFAT inhibition is also necessary. Many undesirable side effects of CNIs are due to calcineurin and NFAT expression in nonimmune cells as well as the diverse roles of NFAT in regulating effector $\mathrm{T}$ cell differentiation (5). Therefore, if it were possible to specifically inhibit TCR-proximal calcineurin, or Lck itself, and if this inhibition were sufficient to control disease, the strategy could provide a valuable alternative to existing CNIs. Interestingly, one study found that transplantation of $\mathrm{T}$ cells deficient in NFAT1 and/or NFAT2 was protective against aGVHD (17). In that study, mice receiving NFAT-deficient $\mathrm{T}$ cells had expanded Treg populations compared with mice receiving WT $\mathrm{T}$ cells, which could explain the apparent discrepancy between those findings and the findings of Otsuka et al. (15). It is also important to note that Otsuka et al. only determined the importance of inhibiting Lck-S59 dephosphorylation for CNI-mediated control of aGVHD (15). Future studies should address whether inhibition of TCR-proximal calcineurin is essential for the effectiveness of CNIs in other contexts, such as solid organ transplantation and autoimmune disease.

Address correspondence to: Joel L. Pomerantz, 623 Miller Research Building, Baltimore, Maryland 21205, USA. Phone: 443.287.3100; Email: joel.pomerantz@ jhmi.edu.

1. Farouk SS, Rein JL. The many faces of calcineurin inhibitor toxicity-what the FK? Adv Chronic Kidney Dis. 2020;27(1):56-66.

2. Gergis U, van Besien K. Tocilizumab, in search for a role in acute GVHD. Leuk Lymphoma. 2019;60(9):2101-2103.

3. Zeiser R, Socié G. The development of ruxolitinib for glucocorticoid-refractory acute graft-versus-host disease. Blood Adv. 2020;4(15):3789-3794.

4. Shlomchik WD. Graft-versus-host disease. Nat Rev Immunol. 2007;7(5):340-352.

5. Lee JU, et al. Revisiting the concept of targeting NFAT to control T cell immunity and autoimmune diseases. Front Immunol. 2018;9:2747.

6. Gijtenbeek JMM, et al. Cyclosporine neurotoxic- ity: a review. J Neurol. 1999;246(5):339-346.

7. Textor SC, et al. Posttransplantation hypertension related to calcineurin inhibitors. Liver Transpl. 2000;6(5):521-530.

8. Nguyen T, Di Giovanni S. NFAT signaling in neural development and axon growth. Int J Dev Neurosci. 2008;26(2):141-145.

9. Macian F. NFAT proteins: key regulators of T-cell development and function. Nat Rev Immunol. 2005;5(6):472-484.

10. Liu J, et al. Calcineurin is a common target of cyclophilin-cyclosporin A and FKBP-FK506 complexes. Cell. 1991;66(4):807-815.

11. Dutta D, et al. Recruitment of calcineurin to the TCR positively regulates T cell activation. Nat Immunol. 2017;18(2):196-204.

12. Gaud G, et al. Regulatory mechanisms in $\mathrm{T}$ cell receptor signalling. Nat Rev Immunol. 2018;18(8):485-497.

13. Wang H, et al. ZAP-70: an essential kinase in T-cell signaling. Cold Spring Harb Perspect Biol. 2010;2(5):1-18

14. Watts JD, et al. Phosphorylation of serine 59 of p56(lck) in activated T cells. J Biol Chem. 1993;268(31):23275-23282.

15. Otsuka $S$, et al. Calcineurin inhibitors suppress acute graft-vs-host disease via NFAT-independent inhibition of $\mathrm{T}$ cell receptor signaling. J Clin Invest. 2021;131(11): 147683.

16. Paster W, et al. A THEMIS: SHP 1 complex promotes T-cell survival. EMBO J. 2015;34(3):393-409.

17. Vaeth M, et al. Selective NFAT targeting in T cells ameliorates GvHD while maintaining antitumor activity. Proc Natl Acad Sci US A. 2015;112(4):1125-1130. 